\title{
Grid-climbing Behaviour as a Pain Measure for Cancer-induced Bone Pain and Neuropathic Pain
}

\author{
SARAH FALK, SIMONE GALLEGO-PEDERSEN and NICOLAS C. PETERSEN \\ Center for Neuroscience, Copenhagen, Denmark
}

\begin{abstract}
Despite affecting millions of people, chronic pain is generally treated insufficiently. A major point of focus has been the lack of translation from preclinical data to clinical results, with the predictive value of chronic pain models being a major concern. In contrast to current focus on stimulus-based nociceptive responses in preclinical research, development of behavioural tests designed to quantify suspension of normal behaviour is likely a more equivalent readout for human pain-assessment tests. In this study, we quantified grid-climbing behaviour as a non-stimulus-evoked behavioural test for potential use as a measure of neuropathic and cancer-induced bone pain in mice. In both models, the grid-climbing test demonstrated pain-related sparing of the affected leg during climbing. In both models, the behaviour was reversed by administration of morphine, suggesting that the observed behaviour was pain-specific.
\end{abstract}

Chronic pain affects up to $40 \%$ of the general population and consequently has a major socio-economic impact on our society $(1,2)$. Despite affecting millions of people, chronic pain is generally insufficiently treated, leaving a large number of patients with inadequate pain-relief $(2,3)$. The low success rate for development of new analgesic therapy has been linked to a poor translation from preclinical findings to clinical effects $(4,5)$, which have led to extensive criticism of the predictive value of the animal models of chronic pain (5-7). However, animal models are currently the best available tool for testing the analgesic potential of new drugs. A way to increase the probability of successful translation could, therefore, be to rethink the way models are used. There is currently an extensive mismatch between preclinical and clinical assessment of pain, and it likely reduces the chances

This article is freely accessible online.

Correspondence to: Sarah Falk, Center for Neuroscience, Blegdamsvej 3B, 2200 Copenhagen N, Denmark. E-mail: falk@sund.ku.dk

Key Words: Pain behaviour, chronic pain, neuropathic pain, cancerinduced bone pain, grid climbing. of translation from preclinical findings to clinical efficacy.

Most preclinical research relies on detection of responses to mechanical or thermal stimuli, hence the evaluation of evoked pain. In contrast, clinical research aims at quantifying the subjective pain experience, mainly evaluated by self-reporting systems, such as the numeric or visual analogue scales (8), and hence quantifying ongoing pain. Consequently, preclinical research is focused on stimulus-based evoked responses, mainly mediated by spinal processes, whereas clinical studies are evaluating the pain experience, including perception at supraspinal levels. Rodents are still the primary choice for in vivo models of various pain conditions, and although tradition has favoured quantification of evoked responses, rodents do display aversive motivational states that are similar to aspects of human pain perception $(9,10)$. Aversive affective functions in animals are likely adaptive survival mechanisms, and are based on the ability to alter behavioural decisions or suspend normal behaviour in order to avoid injury or permit faster healing of a damaged body part. Development of behavioural tests designed to quantify suspension of normal behaviour is, therefore, likely a more relevant readout for human pain assessment, and validation and implementation of such tests might ease the translation from in vivo model to patient.

In this study we tested if sparing of the affected leg during grid climbing behaviour can be used as a non-stimuli-evoked behavioural test to quantify neuropathic-spared nerve injury (SNI) and cancer-induced bone (CIBP) pain. The test is based on suspension of the animal's normal behaviour, and hence reflects how the ongoing pain affects that animal's natural behaviour. In addition, the tests are based on objective measures, which reduces the variability that can be associated with application of a given stimuli and interpretation of responses by the experimenter.

\section{Materials and Methods}

Animals. Six-week-old male and female $\mathrm{C} 3 \mathrm{H} / \mathrm{HeNRj}$ mice (Taconic, Tornbjerg, Denmark) were group-housed in Type III IVC cages (Scanbur A/S, Karlslunde, Denmark) with a 12-hour light/dark cycle, temperature of $21^{\circ} \mathrm{C} \pm 1{ }^{\circ} \mathrm{C}$, a relative humidity of $45-55 \%$ and allowed free access to water and standard diet (Brogaarden Aps, 
Lynge, Denmark). All experiments were approved by the Danish Committee for Experiments on Animals, 2015-15-0201-00678, Copenhagen, Denmark, and conducted according to the guidelines of the International Association for the Study of Pain (11).

Cell line. NCTC clone 2472 cells (ATCC ${ }^{\circledR}$ CCL-11; Boras, Sweden) were kindly provided by Anne-Marie Heegaard, Department of Drug Design and Pharmacology, University of Copenhagen. Cells were cultured in NCTC-135 medium (Sigma-Aldrich, Copenhagen, Denmark) supplemented with sodium hydrogen carbonate and $10 \%$ equine serum (Sigma-Aldrich). On the day of inoculation, cells were harvested at approximately $70 \%$ confluence with $1 \mathrm{mM}$ ethylenediaminetetra-acetic acid in $0.1 \mathrm{M}$ Dulbecco's phosphate buffered saline (D-PBS), and resuspended in 0.1 M D-PBS to a final density of $10^{7}$ cells $/ \mathrm{ml}$. Cancer cells were kept on ice until use.

Model of $C I B P(12)$. Briefly, mice were anesthetized with a mixture of ketamine $(80 \mathrm{mg} / \mathrm{kg})$ and xylazine $(17 \mathrm{mg} / \mathrm{kg})$. Following an incision made in the skin overlying the patella, the lateral site of the patella tendon and lateral retinaculum tendon were loosened and the patella pushed aside to expose the distal femoral epiphysis. A 30gauge needle (Mediq, Brøndby, Denmark) was used to drill a hole into the medullary cavity through which NCTC cells in $10 \mu \mathrm{l}$ NCTC-135 medium (Sigma-Aldrich) were inoculated with a $0.3 \mathrm{ml}$ insulin syringe (Terumo Medical Corporation, Therumo, Herlev, Denmark). The hole was closed with dental cement (Mediq, Brøndby, Denmark) and the open wound thoroughly irrigated with sterile saline. The skin was closed with two wound clips (Mediq, Brøndby, Denmark). Sham-operated control mice underwent the same operation, but were inoculated with medium alone.

Spared nerve injury. The SNI model of neuropathic pain was performed as previously described (13). Briefly, animals were anaesthetized with a mixture of ketamine $(80 \mathrm{mg} / \mathrm{kg})$ and xylazine $(17 \mathrm{mg} / \mathrm{kg})$ and the skin and left thigh muscles incised. The sciatic nerve was carefully exposed and the tibial and common peroneal nerves ligated with $5 / 0$ silk suture and cut distally, while leaving the sural nerve intact. Sham animals underwent the same procedure but without ligating and cutting the nerves.

Post-operative analgesia. All animals received Rimadyl (s.c. $5 \mathrm{mg} / \mathrm{kg}$; Pfizer, Ballerup, Denmark) and 2\% Lidocaine gel (AstraZeneca, Copenhagen, Denmark) was applied to the wound.

Morphine administration. Morphine hydrochloride at $10 \mathrm{mg} / \mathrm{kg}$ (Morfin; Skanderborg Pharmacy, Denmark) dissolved in saline was administered i.p. and the animals were placed in the home cage for $10 \mathrm{~min}$. Animals were then retested in the respective behavioural tests to established post-morphine values.

Humane endpoint. Humane endpoint for animals with CIBP was determined as score 0 for limb use. When scoring 0 , the animal was euthanized and the last measure carried forward for data analysis. Animals with excessive muscular tumours were excluded, as muscular tumours may interfere with the free movement of the leg thereby biasing the behavioural readout.

Behaviour test. Animals were familiarised with the behavioural laboratory and each behavioural test at least twice prior to baseline measures.
Limb use test (14). The mouse was allowed to acclimatize $10 \mathrm{~min}$ in a transparent standard cage without bedding $(350 \mathrm{~mm} \times 450 \mathrm{~mm}$ $\times 185 \mathrm{~mm}$; Scanbur). The animal was subsequently observed for 3 min and a limb use score from 4 to 0 was assigned: 4: normal use of affected limb, 3: insignificant limping, 2: significant limping, 1: significant limping and partial lack of limb use, and 0: total lack of limb use.

Von Frey test. Mechanical hypersensitivity was evaluated by the upand-down method (15). Briefly, mice were placed in plastic containers on a wire mesh floor and allowed to acclimatize for $30 \mathrm{~min}$. Starting at $0.4 \mathrm{~g}$, filaments ranging from 0.04 to $4 \mathrm{~g}$ were applied to the plantar surface of the hind paw with a minimum 3-min interval between two stimulations. A stimulus was recorded as positive if paw withdrawal was observed within $3 \mathrm{~s}$ of stimulation. The two responses detecting the threshold were designated as the first two responses in a series of six. The $50 \%$ response withdrawal threshold was calculated using the formula: $50 \%$ g threshold $=(10 \mathrm{Xf}+\mathrm{k} \delta) /(10,000)$, where $\mathrm{Xf}=$ value (log unit) of the final filament; $k=$ tabular value for the pattern of positive/negative responses; and $\delta=$ mean difference (in log units) between filaments.

Grid climbing test. Each animal was placed on a standard wire lid. The lid was turned upside down with the animal now hanging from underneath for $2.5 \mathrm{~min}$ while being filmed from above. Each film was subsequently analysed in slow-motion and time spent not using the affected leg was recorded manually.

Blinding of experiments. All experiments, and subsequent analyses, were blinded for the experimenters.

Statistical analysis. Statistical analysis was performed with GraphPad Prism (vers. 4.03 for Windows; GraphPad Software, San Diego, CA, USA). All behavioural tests were analysed with repeated two-way ANOVA with a Bonferroni's multiple comparisons test. Pre- and postmorphine values were analysed with a paired two-tailed Student's $t$ test. All data are presented as the mean \pm SEM. For all statistical analyses, a probability value of 0.05 was considered significant.

\section{Results}

Validation of pain condition by standardized behaviour test. Standardized behaviour tests, commonly used for pain quantification in CIBP and SNI, were used to validate successful implementation of the models (4, 16-20). In the CIBP model, scoring of limb use demonstrated a significant decrease in limb use on day 18 post surgery (Figure 1A). 10 $\mathrm{mg} / \mathrm{kg}$ morphine significantly abrogated the decrease in limb use. SNI was evaluated by detection of mechanical hypersensitivity. Mechanical hypersensitivity, demonstrated by a significant decrease in the $50 \%$ withdrawal threshold, was observed on day 18 . Again, $10 \mathrm{mg} / \mathrm{kg}$ morphine significantly abrogated the decrease in withdrawal threshold (Figure 1B).

Grid-climbing behaviour as a measure of pain. Observations of mice in their home cages demonstrated a natural impulse for climbing the wire lids. This behaviour was quantified by 
A

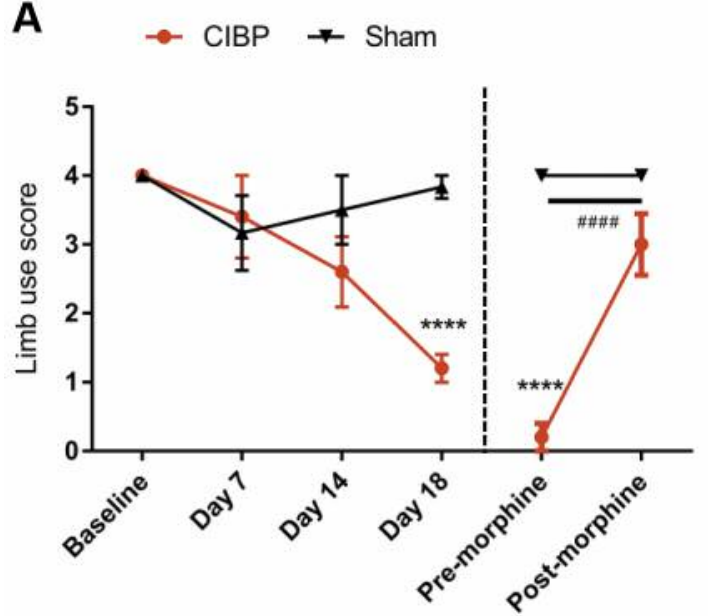

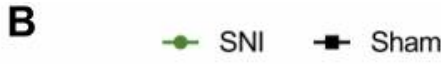

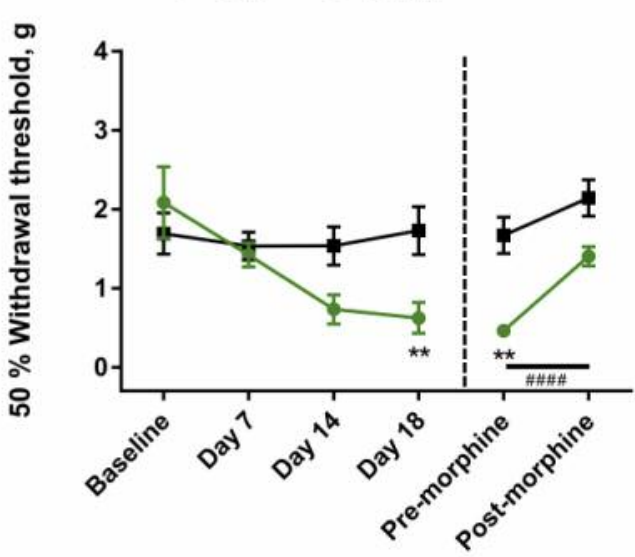

Figure 1. A: The limb use score was significantly lower on day 18 in animals with cancer-induced bone pain (CIBP) compared to sham-treated animals, and significantly improved following administration of $10 \mathrm{mg} / \mathrm{kg}$ morphine. B: The $50 \%$ withdrawal threshold was significantly reduced on day 18 in the group with spared nerve injury (SNI) compared to the sham group, and was significantly increased following administration of 10 $\mathrm{mg} / \mathrm{kg}$ morphine. Data are the mean \pm SEM of $n=5-6$ mice. Significantly different at $* p<0.05$, ** $p<0.01$, *** $p<0.001$, ****p<0.0001 vs. sham, or pre-morphine vs. post-morphine groups.
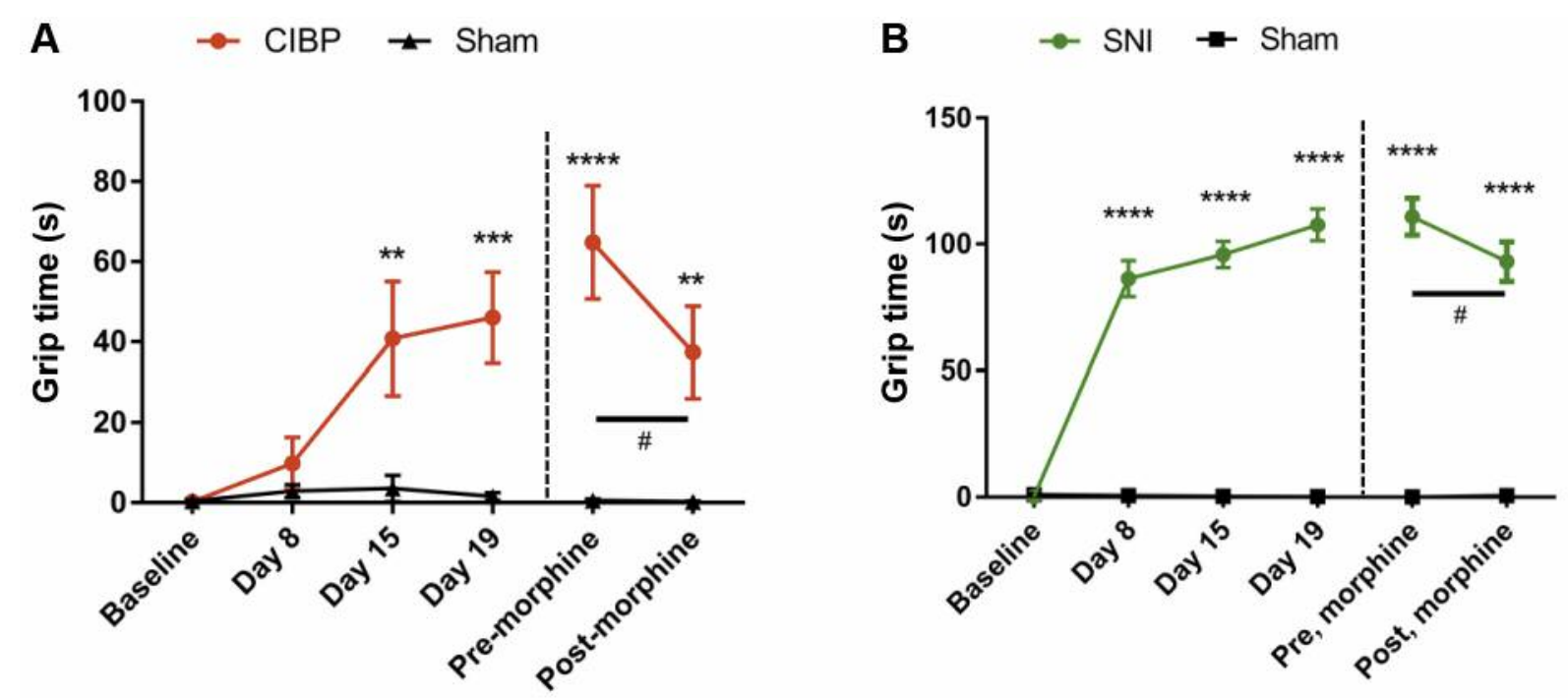

Figure 2. A: Sparing time was significantly increased in animals with cancer-induced bone pain (CIBP) at day 15 and 19, and significantly decreased following administration of $10 \mathrm{mg} / \mathrm{kg}$ morphine. B: Sparing time was significantly increased in the group with spared nerve injury (SNI) from day 8 to 19, and significantly decreased following administration of $10 \mathrm{mg} / \mathrm{kg}$ morphine. Data are the mean \pm SEM of $n=9-10 \mathrm{mice}$. Significantly different at $* p<0.05, * * p<0.01, * * * p<0.001, * * * * p<0.0001$ vs. sham, or pre-morphine vs. post-morphine groups.

measuring the time spent sparing the affected leg during climbing. Sparing time was significantly increased in both models. In animals with CIBP, a significant increase was detected on day 15 (Figure 2A). In addition, sparing time significantly decreased following administration of morphine. In the model of SNI, a significant increase in sparing time was observed from day 8 (Figure 2B). Furthermore, the time spent sparing the leg during climbing was significantly reduced by administration of morphine (Figure 2B).

\section{Discussion}

The lack of translation for preclinical findings to clinical efficacy has been a major issue of discussion. In this study, 
we attempted to bridge the gap between clinical and preclinical pain assessment, by validating a new behavioural test for assessment of pain in two of the most commonly used mouse models of neuropathic pain and CIBP.

In contrast to more traditional preclinical pain assessment, the presented behavioural test is based on suspension of the animal's normal behaviour. Commonly used preclinical pain assessments, such as the von Frey test or Hargreaves test, are generally based on mechanical or thermal stimuli induced by the experimenter $(19,21)$. This approach introduces bias as the read-out is influenced both by the uniformity, or lack thereof, of the applied stimuli, and the subjective interpretation of the response by the experimenter. The gridclimbing test evaluated in this study is, in contrast, based on a behaviour frequently observed in the home cage. Observations of naive animals in their home cage demonstrate that they are fully capable of, and frequently do climb the wire lid with two or three legs. However, as demonstrated by the sham groups, elevating the wire lid 30 $\mathrm{cm}$ from the ground motivates the animals to use all four legs during the majority of the $2.5 \mathrm{~min}$ test period. Suspension of this behaviour was observed in both models, demonstrated as a significant increase in the time sparing the affected leg. In both models, administration of morphine significantly reduced sparing time, suggesting that the observed behaviour is pain-specific, and not only caused by issues related to motor function induced by the surgery or to tumour growth. Interestingly, in the model of CIBP, the significant increase in time sparing the affected leg was detected as early as day 15 , hence earlier than detection of pain behaviour using the standardized limb use test, thereby suggesting a high sensitivity of the grid-climbing test.

In contrast, the data from the SNI model needs a more careful interpretation. Although a significant increase in sparing time was detectable from day 8 , it is clear that at least part of the observed behaviour was caused by motor function abnormalities associated with the damage to the sciatic nerve. The motor function abnormalities linked to introduction of the model have previously prevented assessment of neuropathic pain using gait analysis. In neuropathic models, traditional analgesic compounds, such as morphine and gabapentin, have failed to improve the behavioural readout using standard tool for gait analysis $(22,23)$, thereby suggesting that the observed behaviour was not pain-related but rather caused by motor function abnormalities. However, in this study, a significant decrease in sparing time was induced by administration of morphine, suggesting that the grid-climbing test does in fact quantify pain and not only motor function abnormalities. Using the traditional von Frey test, mechanical hypersensitivity was significant on day 18 , in contrast, significant increased sparing time was detected as early as day 8 . Whether the earlier detection is caused by the associated motor function abnormalities, pain, or a combination of both requires further studies. Still, it should be noted that the inter-animal variation was smaller in the grid-climbing test compared to the von Frey test, suggesting that fewer animals might be required in a given study, which is advantageous from an ethical aspect.

Another advantage of the grid-climbing test is the high objectivity. Assessment of mechanical hypersensitivity requires training of the experimenter and involves a subjective element for both application of the stimuli and detection of a response $(19,21)$. Similarly, assessment of limb use requires training for the experiment. In general, for both methods, all time points in a given study have to be evaluated by the same experimenter. The grid-climbing test is quantified by post-experimental measuring of the behaviour in slow-motion. This permits assessment of the test by multiple experimenters, and allows subsequent quantification of the behaviour by several experimenters independently, or several times if needed, thereby eliminating nearly all subjective interpretation.

Overall, the data suggest that suspension of normal climbing behaviour can be an objective tool for quantification of pain in mouse models of CIBP and neuropathic pain. The test is simple and quick to perform, does not require costly specialised equipment or timeconsuming training, neither of the animals nor of the experimenter. The study suggests that grid climbing is a pain-sensitive behaviour that is comparable to standard stimuli-based tests in terms of detection and quantification of pain. We suggest that using suspension of normal behaviour to quantify pain might be a more clinically relevant read-out. The mismatch between evaluation of evoked and ongoing pain in pre-clinical and clinical settings might partly explain the lack of translation from preclinical analgesic effect to pain relief in patients. In the future, implementation of behavioural tests based on suspension of normal behaviour might help ease this translation.

\section{Conflicts of Interests}

The Authors declare that there is no conflict of interest in regard to this study.

\section{References}

1 van Hecke $\mathrm{O}$, Torrance $\mathrm{N}$ and Smith $\mathrm{BH}$ : Chronic pain epidemiology and its clinical relevance. Br J Anaesth 111(1): 13$18,2013$.

2 Breivik H, Eisenberg $\mathrm{E}$ and O'Brien T: The individual and societal burden of chronic pain in Europe: the case for strategic prioritisation and action to improve knowledge and availability of appropriate care. BMC Public Health 13: 1229, 2013.

3 Greco MT, Roberto A, Corli O, Deandrea S, Bandieri E, Cavuto $\mathrm{S}$ and Apolone G: Quality of cancer pain management: an update of a systematic review of undertreatment of patients with cancer. J Clin Oncol 32(36): 4149-4154, 2014. 
4 Gregory NS, Harris AL, Robinson CR, Dougherty PM, Fuchs PN and Sluka KA: An overview of animal models of pain: disease models and outcome measures. J Pain 14(11): 1255-669, 2013.

5 Berge OG: Predictive validity of behavioural animal models for chronic pain. Br J Pharmacol 164(4): 1195-1206, 2011.

6 Whiteside GT, Adedoyin A and Leventhal L: Predictive validity of animal pain models? A comparison of the pharmacokineticpharmacodynamic relationship for pain drugs in rats and humans. Neuropharmacology 54(5): 767-775, 2008.

7 Mogil JS, Davis KD and Derbyshire SW: The necessity of animal models in pain research. Pain 151(1): 12-17, 2010.

8 Mao J: Translational pain research: achievements and challenges. J Pain 10(10): 1001-1011, 2009.

9 Navratilova E and Porreca F: Reward and motivation in pain and pain relief. Nat Neurosci 17(10): 1304-1312, 2014.

10 Sneddon LU, Elwood RW, Adamo SA and Leach MC: Defining and assessing animal pain. Animal Behaviour 97: 201-212, 2014

11 Zimmermann M: Ethical guidelines for investigations of experimental pain in conscious animals. Pain 16(2): 109-110, 1983.

12 Falk S, Uldall M, Appel C, Ding M and Heegaard AM: Influence of sex differences on the progression of cancer-induced bone pain. Anticancer Res 33(5): 1963-1969, 2013.

13 Bourquin AF, Suveges M, Pertin M, Gilliard N, Sardy S, Davison AC, Spahn DR and Decosterd I: Assessment and analysis of mechanical allodynia-like behavior induced by spared nerve injury (SNI) in the mouse. Pain 122(1-2): 14e1, 2006.

14 Vermeirsch H, Nuydens RM, Salmon PL and Meert TF: Bone cancer pain model in mice: evaluation of pain behavior, bone destruction and morphine sensitivity. Pharmacol Biochem Behav 79(2): 243-251, 2004.

15 Dixon WJ: Efficient analysis of experimental observations. Annu Rev Pharmacol Toxicol 20: 441-462, 1980.
16 Pacharinsak $\mathrm{C}$ and Beitz A: Animal models of cancer pain. Comp Med 58(3): 220-33, 2008.

17 Currie GL, Delaney A, Bennett MI, Dickenson AH, Egan KJ, Vesterinen HM, Sena ES, Macleod MR, Colvin LA and Fallon MT: Animal models of bone cancer pain: systematic review and meta-analyses. Pain 154(6): 917-26, 2013.

18 Barrot M: Tests and models of nociception and pain in rodents. Neuroscience 211: 39-50, 2012.

19 Chaplan SR, Bach FW, Pogrel JW, Chung JM and Yaksh TL: Quantitative assessment of tactile allodynia in the rat paw. $\mathrm{J}$ Neurosci Methods 53(1): 55-63, 1994.

20 Mogil JS: Animal models of pain: progress and challenges. Nat Rev Neurosci 10(4): 283-294, 2009.

21 Bonin RP, Bories C and De Koninck Y: A simplified up-down method (SUDO) for measuring mechanical nociception in rodents using von Frey filaments. Mol Pain 10: 26, 2014.

22 Mogil JS, Graham AC, Ritchie J, Hughes SF, Austin JS, Schorscher-Petcu A and Langford DJ and Bennett GJ: Hypolocomotion, asymmetrically directed behaviors (licking, lifting, flinching, and shaking) and dynamic weight bearing (gait) changes are not measures of neuropathic pain in mice. Mol Pain 6: 34, 2010.

23 Piesla MJ, Leventhal L, Strassle BW, Harrison JE, Cummons TA, Lu P and Whiteside GT: Abnormal gait, due to inflammation but not nerve injury, reflects enhanced nociception in preclinical pain models. Brain Res 1295: 89-98, 2009.

Received March 20, 2017

Revised April 12, 2017

Accepted April 19, 2017 